\section{SART 3.5D - Recovering cerebrovascular hemodynamics from standard 3D digital subtraction angiography cone-beam CT data-sets}

Sara El Hadji, ${ }^{1}$ Elena De Momi, ${ }^{1}$ Francesco Cardinale, ${ }^{2}$ Giuseppe Baselli ${ }^{1}$

${ }^{1}$ Department of Electronics, Information and Bioengineering, Politecnico di Milano; ${ }^{2}$ Claudio Munari Centre for Epilepsy and Parkinson surgery, Niguarda Ca' Granda Hospital, Milano, Italy

\section{Background}

Brain vasculature visualization is gaining more and more importance in neuroimaging and neurosurgery in both pre-operative and intra-operative phases. The contrast dynamics through the various districts can be informative for various pathologies and also provides keys to distinguish arteries and veins $(\mathrm{A} / \mathrm{V})$ in surgical planning. Further light is also shed over the dynamic feature of arterial and venous cerebral flow phases.

\section{Method}

SART $3.5 \mathrm{D}^{1}$ is a modified simultaneous algebraic reconstruction technique (SART) ${ }^{2}$ which post- processes the contrast enhanced (CE) cone-beam CT (CBCT) data ${ }^{3}$ extracting the contrast medium (CM) dynamics in the previously segmented 3D angiography. Voxel wise Time Intensity Curves (TICs) are modelled as a linear combination of temporal basis functions. The voxel-wise $\mathrm{A} / \mathrm{V}$ classification require the venous area under the curve $\left(A U C_{V}\right)$ to be computed, after the subject specific tuning of A/V separation time based on clustering.

\section{Results}

Ten data-sets were considered for the validation against manually annotated
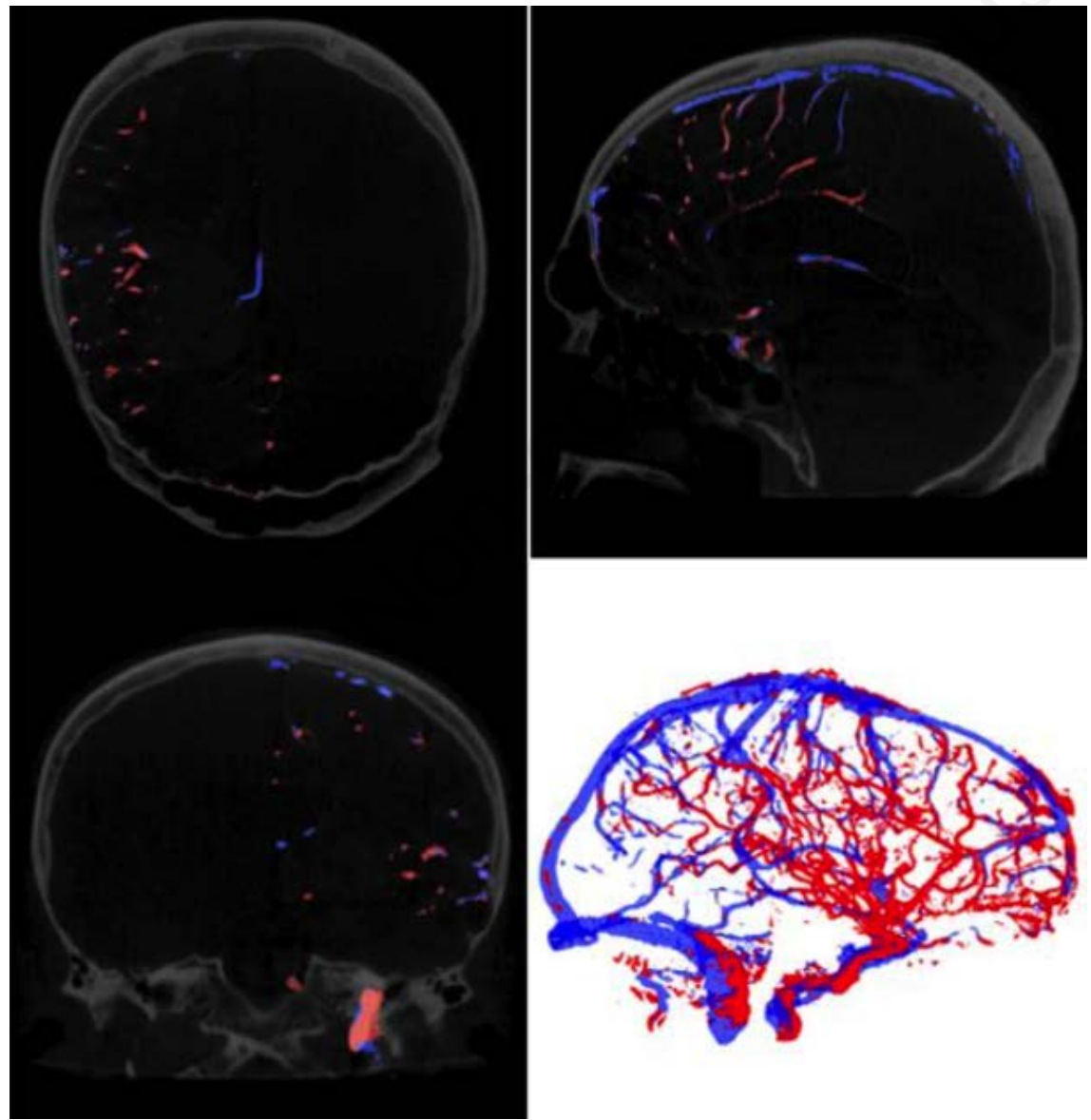

Figure 1. Example of classification of arteries (red) and veins (blue). Bottom-Left) DSA with O-armTM CB-CT for stereo-EEG planning labelled. The first three panels report the sagittal, coronal and axial view of a 3D CE-mask with the voxels labelled as arteries or veins in red and blue, respectively. The latter represent a volume rendering of the labelled 3D DSA.
Correspondence: Sara El Hadji, Department of Electronics, Information and Bioengineering, Politecnico di Milano, Milano, Italy.

E-mail: sara.elhadji@polimi.it

Key words: Neurovascular diseases; meeting.

Conference presentation: $9^{\text {th }}$ Annual Meeting of the International Society of Neurovascular Disease (ISNVD), May $30^{\text {th }}-31^{\text {st }}, 2019$, Ferrara, Italy.

This work is licensed under a Creative Commons Attribution 4.0 License (by-nc 4.0)

(C) Copyright: the Author(s), 2019

Licensee PAGEPress, Italy

Veins and Lymphatics 2019; 8:8427

ROIs. The overall statistics (median and interquartile range) is reported below. Sensitivity has a median value of 0.91 [0.82-0.93] (first and third quartile in brackets). Specificity has a median value of 0.82 [0.74-0.92]. While for the accuracy, a median value of 0.85 was obtained with an IQ range [0.82-0.86].

\section{Conclusions}

Results on clinical data confirmed applicability and robustness of the method (Figure 1). Moreover, possible extension of the method for a time-resolved 3D angiography might come from a $\mathrm{CM}$ arrival time surrogate given by $1-\mathrm{AUCV}_{\mathrm{V}} / \mathrm{AUC}$. Future improvement considers the inclusion of spatial continuity criteria for a spatio-temporal classifier.

\section{References}

1. El Hadji S, Bonilauri A, De Momi E, et al. Automatic arteries and veins detection from $3 \mathrm{D}$ brain $\mathrm{CBCT}$ angiographies. A preliminary study. In GNB 2018 Sixth National Congress of Bioengineering, June 2018, Politecnico di Milano, Milan, Italy.

2. Andersen AH, Kak AC. Simultaneous algebraic reconstruction technique (SART): a superior implementation of the ART algorithm. Ultrason Imaging 1984;6:81-94.

3. Cardinale F, Pero G, Quilici L, et al. Cerebral angiography for multimodal surgical planning in epilepsy surgery: description of a new three- dimensional technique and literature review. World Neurosurg 2015;84:358-67. 